\title{
Elastography for Predicting and Localizing Nodal Metastases during Endobronchial Ultrasound
}

\author{
Takahiro Nakajima $^{a}$ Terunaga Inage ${ }^{a}$ Yuuki Sata ${ }^{a}$ Junichi Morimoto ${ }^{a}$ \\ Tetsuzo Tagawa $^{a}$ Hidemi Suzuki $^{a}$ Takekazu Iwata ${ }^{a}$ Shigetoshi Yoshida ${ }^{a}$

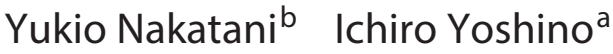 \\ Departments of ${ }^{\mathrm{a}}$ General Thoracic Surgery and ${ }^{\mathrm{b}}$ Diagnostic Pathology, Graduate School of Medicine, Chiba \\ University, Chiba, Japan
}

\section{Key Words}

Endobronchial ultrasound · Lung cancer imaging ·

Diagnostic bronchoscopy · Elastography

\begin{abstract}
Background: Elastography is a relatively new technology that can generate images reflective of tissue stiffness (elasticity). Neoplastic tissue is usually stiffer than normal structures. Objectives: The aim of this study was to evaluate the feasibility and utility of elastography when combined with convex-probe endobronchial ultrasound (CP-EBUS) for predicting and localizing metastatic lymph nodes during endobronchial ultrasound with transbronchial needle aspiration (EBUS-TBNA). Methods: Consecutive results of endobronchial elastography of lymph nodes performed using EBUSTBNA were prospectively collected and retrospectively analyzed. Elastography images were acquired as JPEG images and also recorded as video clips. Stiff area ratios [(stiff areas as blue pixels) / (lymph node areas as region of interest pixels)] for each lymph node determined by elastography were collated with the results of pathological diagnosis. We also performed elastography of surgically resected lymph nodes
\end{abstract}

and compared image findings with pathological sections. Results: We evaluated 49 lymph nodes in 21 patients by CPEBUS. There were 16 metastatic nodes (10 lung cancer metastases and 6 metastases from extrathoracic malignancies). Mean stiff area ratios were significantly greater for metastatic lymph nodes (0.478) than for benign nodes $(0.216 ; p=$ 0.0002 ). Using a cutoff value of 0.311 for stiff area ratios, the sensitivity and specificity for predicting metastatic disease were 0.81 and 0.85 , respectively. The stiff area was histologically compatible with metastatic distribution in surgically resected lymph nodes. Conclusions: Endobronchial elastography is feasible for lymph nodes when combined with CPEBUS. Stiff area ratios are useful for predicting metastatic lymph nodes, which may be an efficient guide for TBNA.

(c) 2015 S. Karger AG, Basel

\section{Introduction}

Neoplastic tissue usually shows higher cellularity and vascularity, which results in stiffer tissue, compared to normal structures [1]. The idea to use tissue stiffness as a novel diagnostic imaging tool originated in breast cancer

\section{KARGER 125}

(C) 2015 S. Karger AG, Basel

0025-7931/15/0906-0499\$39.50/0

E-Mail karger@karger.com

www.karger.com/res
Takahiro Nakajima, MD, PhD, FCCP, Assistant Professor

Department of General Thoracic Surgery

Graduate School of Medicine, Chiba University

1-8-1 Inohana, Chuo-ku, Chiba, 260-8670 (Japan)

E-Mail takahiro_nakajima@med.miyazaki-u.ac.jp 
diagnosis [2]. The initial technology of imaging elasticity was first reported in 1991, and was used to visualize the tissue strain that is caused by compression of the probe [3]. The correlation between stiffness and tissue condition has been well analyzed in the breast [4], and elastography has been commonly used for the diagnosis of breast cancer in the past 5 years [5].

Elastography is a strain imaging technique that assesses tissue stiffness and visualizes the distribution of stiffness in the region of interest (ROI). Tissue stiffness is estimated by measuring the strain of the tissue in response to mechanical stress, either local compression or vibration. To measure the amount of change before and after the ROI is compressed, we can calculate the tissue stiffness. The feedback on tissue stiffness is converted into a color-coded image and displayed in color on an ultrasound image; areas of stiffness can, for example, appear blue and soft areas red.

Elastography in combination with endoscopic ultrasound (transesophageal approach) has been shown to be useful for differentiating between benign and malignant paraesophageal, mediastinal, and abdominal lymph nodes (LNs) [6, 7]. Preliminary data on elastography combined with convex-probe endobronchial ultrasound (CP-EBUS; transbronchial approach) has recently been reported [8]. Izumo et al. [9] subjectively classified EBUS elastography into 3 categories: (1) predominantly non-blue, (2) partly blue, partly non-blue, and (3) predominantly blue. They defined type 3 as malignant and reported that $94.6 \%$ of type 3 LNs showed metastases.

The aim of this study was to evaluate the feasibility and utility of elastography when combined with CP-EBUS for predicting and localizing metastatic LNs during endobronchial ultrasound with transbronchial needle aspiration (EBUS-TBNA).

\section{Materials and Methods}

\section{Study Subjects}

From May 2013 to June 2013, CP-EBUS images (B-mode) as well as endobronchial elastography images were collected from patients with mediastinal and/or hilar adenopathy who underwent EBUS-TBNA for tissue diagnosis. Elastography was observed following B-mode observation. Patients who were diagnosed with or suspected of having lymphoproliferative disease or sarcoidosis were excluded. EBUS-TBNA was performed in patients with mediastinal and/or hilar LNs with a short axis of $\geq 5 \mathrm{~mm}$ on EBUS images following the standard protocol [10].

We also observed surgically removed LNs obtained during radical surgery for lung cancer using the same instruments. Patients with non-small cell lung cancer who underwent thoracotomy for curative intent were enrolled in this analysis.

The definition of each LN location and cancer stage was based on the 7th edition of the TNM classification for lung cancer [11, 12]. This study was approved by the Institutional Review Board of Chiba University, Graduate School of Medicine (approved No. 1664).

\section{Study Design}

We retrospectively reviewed the images that were stored in the JPEG format as well as recorded video clips in the MPEG-4 format. The LN image with the largest diameter was defined as representative. We confirmed that the image was adequately captured by checking the recorded video clip. Then, the images were collated with pathological results obtained by EBUS-TBNA. In this study, we calculated the diagnostic yield, such as sensitivity and specificity, based on EBUS-TBNA results. A negative result was followed by surgical pathology or radiology for more than 6 months.

\section{Endobronchial Elastography}

EBUS procedures were performed on an outpatient basis in patients under conscious sedation (midazolam) with local anesthesia. The CP-EBUS (BF-UC260FW; Olympus Ltd., Tokyo, Japan) equipped with a linear probe on its tip was used to perform EBUS-TBNA. The ultrasound image was processed with a universal endoscopic ultrasound scanner (EU-Y0008, Olympus) at a frequency of $10 \mathrm{MHz}$. Before elastography observation, conventional B-mode observation was performed, and the long and short axes of the LN were measured. Both B-mode and elastography images were captured as JPEG images at the maximum diameter of the LN. The images were reviewed by two individuals (T.N., T.I.).

\section{Elastography for Surgically Removed LNs}

We performed elastography of surgically removed LNs in a completely separate group of patients to compare image findings and the pathologic distribution of metastatic tumor cells. Surgically removed LNs were soaked in normal saline, and B-mode as well as elastography images were obtained using the CP-EBUS instrument. To obtain an elastography image, we applied manual compression and vibration to the LNs. The directions of the observed LNs were marked and cut to make a bisection at the time of elastography. Then, we embedded the LN in the cassette to make a paraffin-embedded section at the same angle as the ultrasound image. The pathological section was stained with hematoxylin and eosin. The loupe image was captured as a JPEG image and the pathologists (J.M., Y.N.) notified us about any metastatic areas in the image. Next, we compared the distribution of cancer metastases within the LN with elastic distribution on the elastography image. We (T.N., T.I.) judged the concordance based on the size and shape of the metastatic area subjectively.

\section{Image Analysis}

The captured images as well as video clips were sent to an independent individual (Y.S.) for image analysis. The following features of the captured image were confirmed: (1) whether the image was captured at the maximum diameter of the LN and (2) whether the characteristics of the elastography image were similar at differ- 


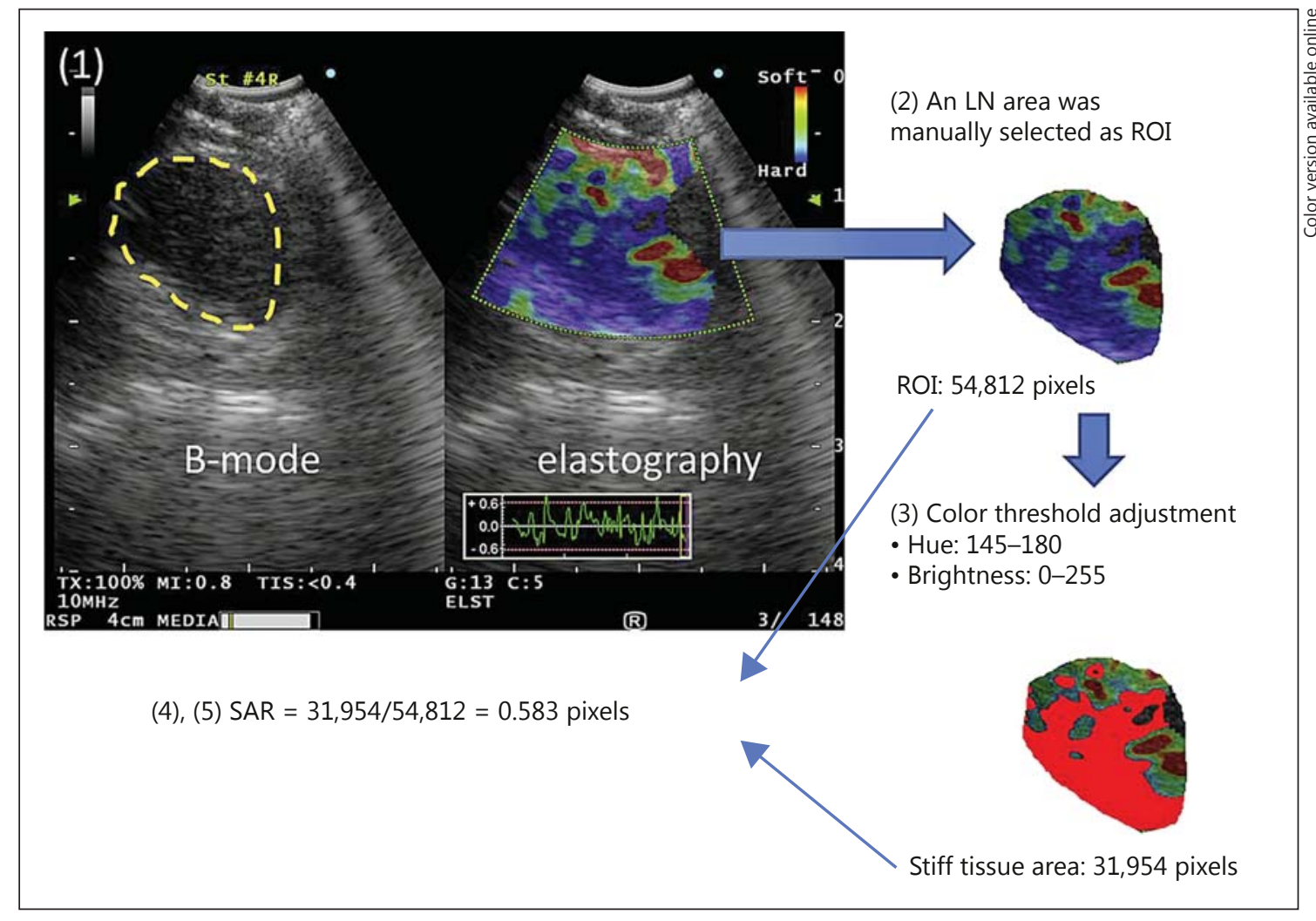

Fig. 1. Quantification of the SAR using ImageJ. For the quantitative analysis of stiffer areas (blue area) within an $\mathrm{LN}$, we defined the SAR as the relatively stiffer area (blue pixel area) per ROI (LN area) on an endobronchial elastography image. For colors, see online version.

ent times (reproducibility) compared to the video clip. In some cases, images were captured at more than two time points. The images were analyzed using dedicated software following the color threshold definition blinded to the pathological results of EBUSTBNA. The pathological section was then also reviewed by two independent individuals (J.M., Y.N.) blinded to the results of elastography.

\section{Quantification of the Stiff Area Ratio}

Elastography allowed the visualization of relative elasticity differences, and stiffer areas were shown in blue in the ultrasound images. For the quantitative analysis of stiffer areas within LNs, we defined the stiff area ratio (SAR; i.e., the percent stiffer tissue area) as stiff area (blue color pixel area) per LN area (ROI) on the elastography image. The LN area (ROI) was selected to cover the entire targeted LN area based on the B-mode image, which was shown beside the elastography image (fig. 1). The SAR was determined with ImageJ $1.45 \mathrm{~s}$ software (National Institutes of Health, Bethesda, Md., USA) using the following image-processing steps (fig. 1): (1) a binary image was created from the raw image; (2) an LN area was manually selected as the ROI; (3) the stiffer tissue area that was visualized as a colored pixel area was determined within a certain threshold level (the same level for all sections: hue: 145-180, brightness: 0-255); (4) the ImageJ measure function was used to determine both the ROI area and the stiffer tissue area (red pixels), and (5) the percentage of the stiffer tissue area of the ROI was calculated. In cases with multiple images, we calculated the mean SAR of each image.

\section{EBUS-TBNA Procedure and Surgical Pathology}

EBUS-TBNA was performed on the LNs observed by elastography. EBUS-TBNA procedures were carried out in the same manner as previously described [10]. Rapid on-site cytology was performed during EBUS-TBNA. We also examined the elastography and pathology of surgically resected LNs in patients with lung cancer. An independent pathologist (Y.N.) reviewed all cases and pathologically confirmed the presence or absence of non-small cell lung cancer cells in each specimen.

\section{Data Analysis}

All elastography images of LNs were compared to the final pathological/clinical results. The sensitivity, specificity, positive predictive value, negative predictive value, and diagnostic accuracy rate were calculated by standard definitions. The pathological results of EBUS-TBNA were considered as the gold standard in this study. Statistical comparisons were made using the MannWhitney $U$ test. General data analysis was conducted using JMP 8.0 software (SAS Institute Inc., Cary, N.C., USA). All p values were based on a two-sided hypothesis, with p values $<0.05$ considered as statistically significant. 
Table 1. Characteristics of the enrolled patients who underwent EBUS-TBNA and their primary diagnosis

\begin{tabular}{lc}
\hline Patients & 21 \\
Male & 15 \\
Female & 6 \\
Age, years & \\
Average & 63 \\
Range & $30-80$ \\
Lung cancer & 14 \\
Adenocarcinoma & 7 \\
Squamous cell carcinoma & 5 \\
Large cell carcinoma & 1 \\
Combined small cell carcinoma & 1 \\
Extrathoracic malignancies & \\
Thymic cancer & 1 \\
Breast cancer & 1 \\
Uterine cervix cancer & 1 \\
Colon cancer & 1 \\
Laryngeal cancer & 1 \\
Unknown primary cancer & 1 \\
Benign adenopathy & \\
Adenopathy, unknown cause & 1 \\
\hline
\end{tabular}

\section{Results}

\section{Patients and Evaluated Samples}

We evaluated 49 LNs in 21 patients by CP-EBUS and performed EBUS-TBNA. Overall, 33 mediastinal and 16 hilar LNs were analyzed in this study. The LN stations that were accessible by CP-EBUS were observed by elastography, and the locations of the LNs did not preclude observation by elastography. There were no station $3 p$ and $2 \mathrm{~L}$ nodes in this case series because of an indication of EBUS-TBNA. In total, 16 metastatic nodes (10 lung cancer metastases and 6 metastases from extrathoracic malignancies including 3 uterine cancers, 2 laryngeal cancers, and 1 breast cancer) were observed. The characteristics of patients and nodes are shown in tables 1 and 2 , respectively.

\section{SAR Analysis in Metastatic LNs}

The characteristics of metastatic LNs which were sampled by EBUS-TBNA are shown in table 3. The SAR was significantly greater for metastatic LNs: 0.487 for metastatic nodes and 0.216 for benign nodes $(p=0.0002$, Mann-Whitney U test; fig. 2). Using a cutoff value of 0.311 for the SAR, the sensitivity and specificity for predicting metastatic disease were 0.81 and 0.85 , respectively (fig. 3).
Table 2. Characteristics of evaluated LNs assessed by EBUS-TBNA

\begin{tabular}{lc}
\hline Lymph nodes $(n=49)$ & \\
Mediastinum & 3 \\
$\quad$ Station 2R & 12 \\
$\quad$ Station 4R & 7 \\
$\quad$ Station 4L & 11 \\
$\quad$ Station 7 & \\
Hilum & 2 \\
$\quad$ Station 10 & 12 \\
$\quad$ Station 11 & 2 \\
$\quad$ Station 12 & \\
\hline Malignant nodes & 5 \\
Adenocarcinoma & 4 \\
Squamous cell carcinoma & 1 \\
Large cell carcinoma & 3 \\
Uterus cervix cancer & 2 \\
Laryngeal cancer & 1 \\
Breast cancer & $8.44(5.0-21.7)$ \\
\hline Average LN size, mm & $7.87(5.0-21.7)$ \\
Benign & $9.60(5.3-16.2)$ \\
Malignant &
\end{tabular}

Figures in parentheses are ranges.

In this study, one false-negative LN was detected at follow-up CT 3 months after EBUS-TBNA. A repeat EBUS-TBNA for this LN revealed lung cancer metastasis (squamous cell carcinoma). The SAR of this LN was 0.456 , which was more than the cutoff value of 0.311 . If we took into consideration this false-negative result, the cutoff value would be 0.311 for the SAR, and the sensitivity and specificity for predicting metastatic disease would be 0.82 and 0.88 , respectively.

\section{Distribution of Metastases in Removed LNs}

Twenty-one patients with non-small cell lung cancer underwent thoracotomy during this study period, and 48 LNs were assessed in this study. Histologies included 13 adenocarcinomas, 4 squamous cell carcinomas, 2 carcinoid tumors, 1 pleomorphic carcinoma, and 1 large cell carcinoma. There were 12 (25\%) metastatic LNs. Overall, 10 out of 12 metastatic LNs (83\%) showed a similar distribution of the metastatic area between the elastography image and the pathological image; the metastatic area displayed a 'relatively hard' sign, shown as blue to green in the elastography image, and the normal LN exhibited a 'relatively soft' sign, shown as yellow to red in the elastography image (fig. 4). 
Table 3. Characteristics of metastatic LNs assessed by EBUS-TBNA

\begin{tabular}{lllll}
\hline Patient No. & Sampled station & Primary diagnosis & SAR & EBUS-TBNA pathology \\
\hline 1 & 7 & Lung cancer & 0.933 & Large cell carcinoma \\
\hline 2 & 7 & Lung cancer & 0.477 & Adenocarcinoma \\
\hline 3 & $4 \mathrm{R}$ & Lung cancer & 0.219 & Adenocarcinoma \\
& $11 \mathrm{~s}$ & & 0.394 & Adenocarcinoma \\
\hline 4 & $4 \mathrm{~L}$ & Lung cancer & 0.183 & Squamous cell carcinoma \\
& $4 \mathrm{R}$ & & 0.583 & Squamous cell carcinoma \\
\hline 6 & $2 \mathrm{R}$ & Lung cancer & 0.434 & Adenocarcinoma \\
\hline 7 & $4 \mathrm{~L}$ & Lung cancer & 0.42 & Adenocarcinoma \\
\hline 8 & $11 \mathrm{i}$ & Lung cancer & 0.56 & Squamous cell carcinoma \\
\hline 9 & $11 \mathrm{~s}$ & Lung cancer & 0.118 & Squamous cell carcinoma \\
\hline 10 & $11 \mathrm{i}$ & Extrathoracic malignancy & 0.827 & Breast cancer \\
& $4 \mathrm{~L}$ & Extrathoracic malignancy & 0.431 & Laryngeal cancer \\
& $4 \mathrm{R}$ & & 0.636 & Laryngeal cancer \\
\hline$(11)$ & 7 & Extrathoracic malignancy & 0.681 & Uterus cervix cancer \\
& $10 \mathrm{~L}$ & & 0.591 & Uterus cervix cancer \\
& $11 \mathrm{~L}$ & & 0.311 & Uterus cervix cancer \\
\hline & 7 & Lung cancer & 0.456 & False negative (SqCC) \\
\hline
\end{tabular}

SqCC $=$ Squamous cell carcinoma

\section{Discussion}

In clinical practice, elastography provides visual information converted from quantitative imaging of the strain and the elastic modulus distributions. Elastic modulus is calculated by measuring the tendency of an object to deform along an axis when tissue is compressed. Malignant tissues tend to be stiffer than normal tissues due to the increased density of tumor cells and vascular structures, as well as fibrosis; therefore, this technology is used to assess various organs for the presence of malignancy [4]. This technology has been integrated with endoscopic devices. Endoscopic ultrasound elastography has been used for differentiating benign and malignant LNs in the mediastinum and abdominal cavity [5].

We frequently encounter multiple LNs within the same station. Furthermore, we need to evaluate multiple stations to properly stage lung cancer. Image guidance technology for predicting suspicious LNs should be useful for efficient nodal staging using EBUS- and endoscopic ultrasound-guided biopsy. Subjective categorizations of B-mode images are useful for excluding negative nodes [13]; however, firm categorization and prediction of negative nodes requires proper training and experience. While the vascular pattern can predict a malignant $\mathrm{LN}$,

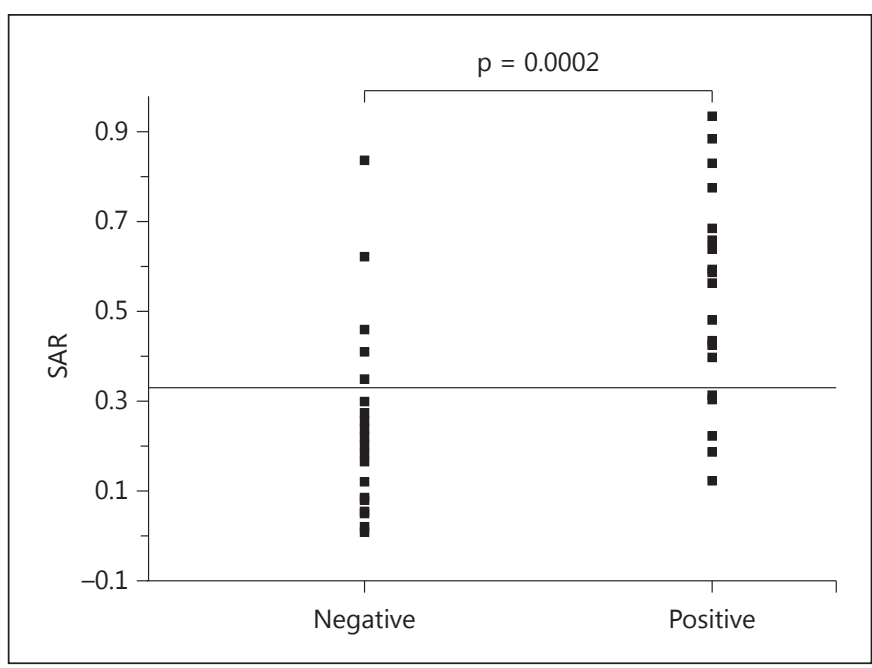

Fig. 2. The SAR of benign and malignant LNs. The SAR was significantly greater for metastatic LNs: 0.478 for metastatic nodes and 0.216 for benign nodes ( $\mathrm{p}=0.0002$, Mann-Whitney $U$ test).

vascular pattern classification is also based on subjective categorization [14]. Therefore, an objective technology for image evaluation to support EBUS- and endoscopic ultrasound-guided biopsy is still required. 


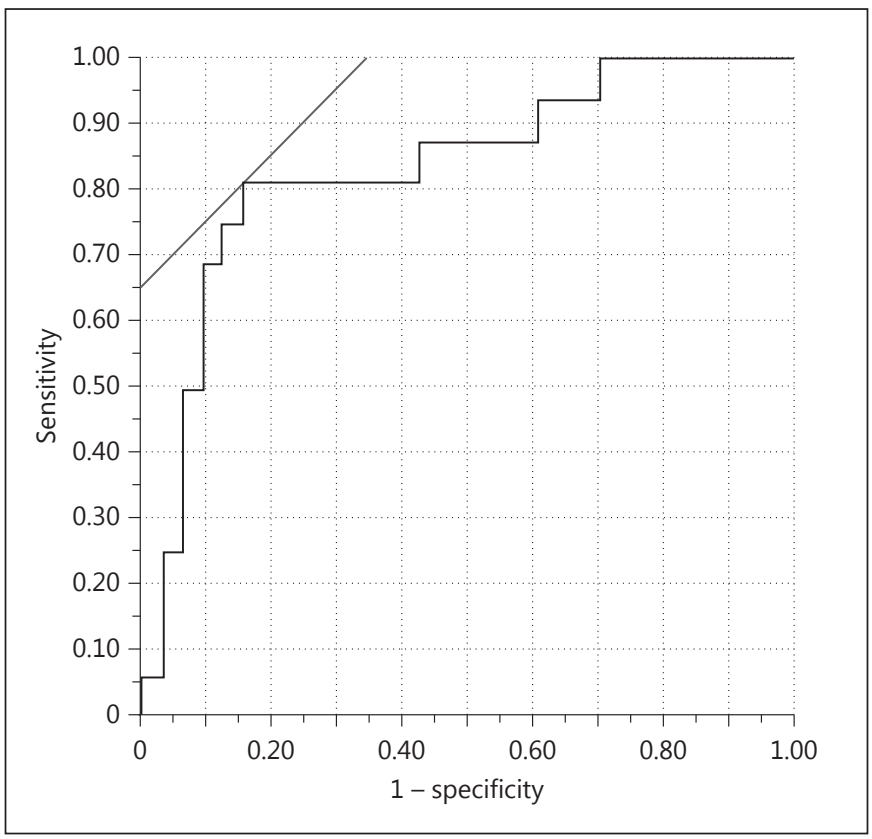

Fig. 3. ROC curve using a cutoff value of 0.311 for SARs. The sensitivity and specificity for predicting metastatic disease were 0.81 and 0.85 , respectively, when the cutoff value was set at 0.311 of the SAR.
We have recently reported the utility of endobronchial elastography for the assessment of tumor invasion to the surrounding structures [15]. The location of each structure is clearly shown in the colored image, and the border between tumor and surrounding tissue can easily be identified due to the color coding used to depict different degrees of tissue stiffness. This efficacy of elastography can be applied to nodal staging during ultrasoundguided needle biopsy procedures. In some cases, the metastatic area was localized within the LN [16]. In such a case, we may puncture the non-cancerous area within the node, yielding a false-negative result. To visualize the suspicious area within an $\mathrm{LN}$, we can control the puncture area within the node and may improve the diagnostic efficacy of EBUS- and endoscopic ultrasound-guided biopsy.

Limitations of this study were that it was a retrospective analysis and the results were based on a small number of samples. The gold standard of this study was pathological results of EBUS-TBNA, because this study intended to evaluate the utility of elastography during EBUS-TBNA. Although one false-negative result was obtained, the SAR was larger than the cutoff value that

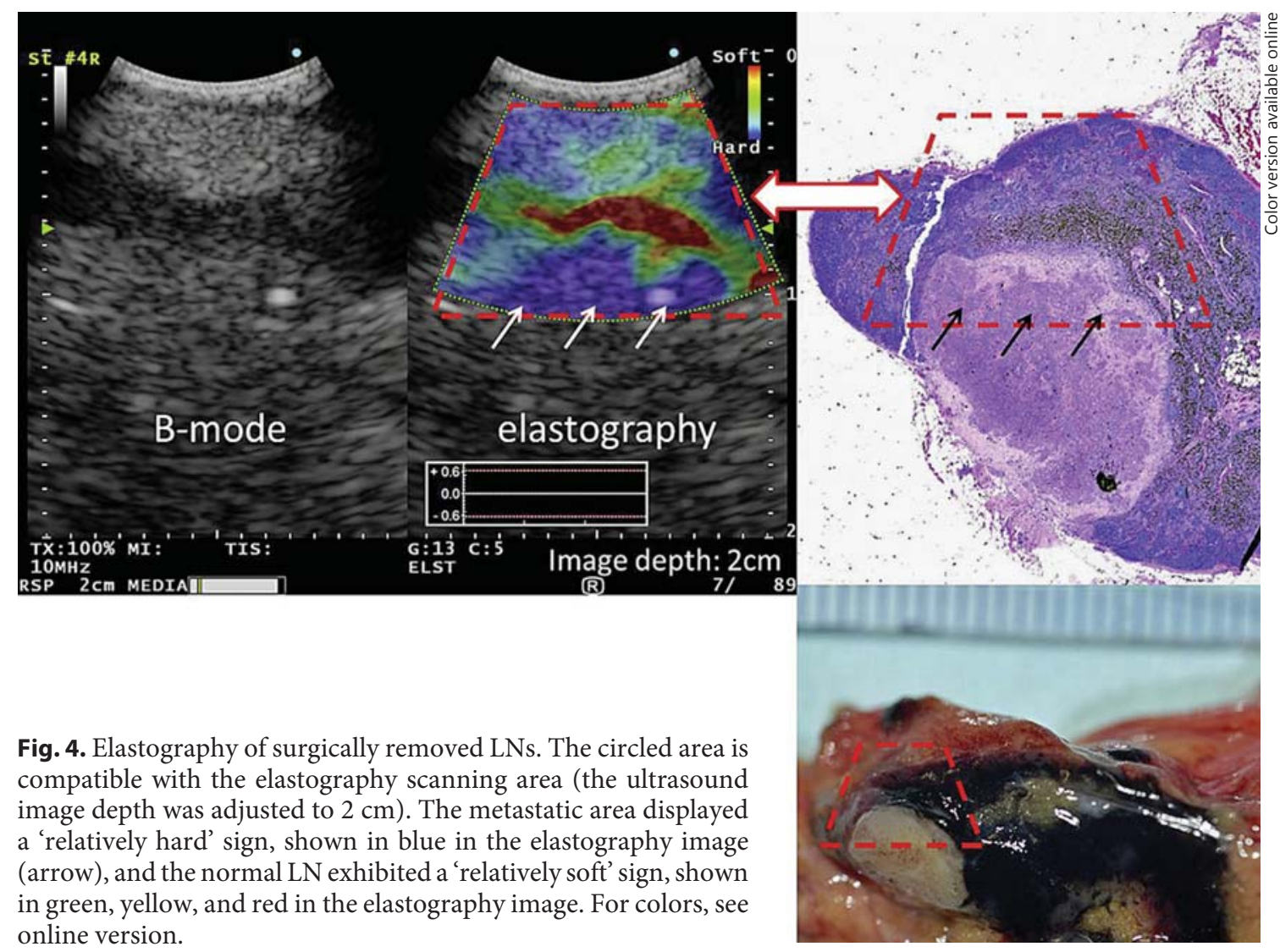


we set in this study. In addition, we did not limit enrollment criteria for lung cancer patients; therefore, some patients with metastatic LNs had extrathoracic malignancies. However, the elastic changes observed in metastatic areas are expected to be similar in other types of cancer tissue compared to normal tissue. An additional limitation was that for surgically removed LNs, the comparison between elastography and pathology was performed by subjective observation and comparison. Similarity analysis between elastography and pathology was also not confirmed objectively. However, we made the pathological section at the same dimension as the elastography image and compared the two images at the same size and angle. Furthermore, the judgment of similarity was made by consensus of two independent researchers.

In the ex vivo analysis, there was no significant difference in the SAR between metastatic and benign LNs. The mean SAR was 0.300 and 0.269 for metastatic and benign LNs, respectively ( $p=0.878$, Mann-Whitney U test). Elastography does not visualize the absolute value of elasticity, and it shows relative stiffness compared with surrounding tissues within the ROI. In the ex vivo analysis, the surgically removed node was held, soaked in normal saline, and then observed by elastography. Hence, the surrounding environment was completely different from the in vivo condition. In this condition, it was hard to select the ROI to cover the whole LN area, and it would make it difficult to evaluate the SAR in the ex vivo condition. The SAR evaluation may not be applicable for the ex vivo evaluation.

\section{Conclusion}

Elastography technology is feasible for mediastinal and hilar LNs via a transbronchial approach using CPEBUS. The SAR may predict nodal metastases and can be used as an objective indicator during EBUS-TBNA. The prediction of nodal metastases using elastography may be a good help to select the LN which should be evaluated as the first priority. This technology may be used to localize metastatic disease within LNs, and real-time elastography guidance for EBUS-TBNA may improve the diagnostic yield as it allows for a precise puncture of the suspicious area within the LN. A prospective study should be conducted to confirm the utility of elastography for the prediction and localization of nodal metastasis in the staging of lung cancer during EBUS-TBNA.

\section{Acknowledgement}

We thank Ms. Fumie Saegusa (Cytotechnologist, Chiba University Hospital) for her support in rapid on-site cytological evaluation. This research is supported by a JFE (the Japanese Foundation for Research and Promotion of Endoscopy) Grant for data collection, analysis, and preparation of the manuscript.

\section{Financial Disclosure and Conflicts of Interest}

Takahiro Nakajima received honoraria and lecture fees from Olympus Medical Systems for EBUS-TBNA training courses. All of the other authors declare that there are no conflicts of interest related to this article. The ultrasound scanner (EU-Y0008) was approved for clinical use by the Ministry of Health, Labour and Welfare in Japan. We leased the EU-Y0008 from Olympus Medical Systems (Tokyo, Japan).

\section{References}

$>1$ Krouskop TA, Wheeler TM, Kallel F, Garra BS, Hall T: The elastic moduli of breast and prostate tissues under compression. Ultrason Imaging 1998;20:260-274.

$>2$ Ueno E, Tohno E, Soeda S, Asaoka Y, Itoh K, Bamber JC, Blaszçzyk M, Davey J, Mckinna JA: Dynamic tests in real-time breast echography. Ultrasound Med Biol 1988;14(suppl 1):53-57.

3 Ophir J, Céspedes I, Ponnekanti H, Yazdi Y, Li X: Elastography: a quantitative method for imaging the elasticity of biological tissues. Ultrason Imaging 1991;13:111-134.

Elastography of Lymph Nodes during EBUS
4 Samani A, Zubovits J, Plewes D: Elastic moduli of normal and pathological human breast tissues: an inversion-technique-based investigation of 169 samples. Phys Med Biol 2007;52: 1565-1576.

5 Balleyguier C, Canale S, Ben Hassen W, Vielh P, Bayou EH, Mathieu MC, Uzan C, Bourgier C, Dromain C: Breast elasticity: principles, technique, results: an update and overview of commercially available software. Eur J Radiol 2013;82:427-434.

-6 Dietrich CF, Saftoiu A, Jenssen C: Real time elastography endoscopic ultrasound (RTEEUS), a comprehensive review. Eur J Radiol 2014;83:405-414.
7 Xu W, Shi J, Zeng X, Li X, Xie WF, Guo J, Lin $\mathrm{Y}$ : EUS elastography for the differentiation of benign and malignant lymph nodes: a metaanalysis. Gastrointest Endosc 2011;74:10011009.

8 Trosini-Désert V, Jeny F, Taillade L, Vignot S, Zribi H, Capron F, Similowski T: Bronchial endoscopic ultrasound elastography: preliminary feasibility data. Eur Respir J 2013;41: 477-479.

-9 Izumo T, Sasada S, Chavez C, Matsumoto Y, Tsuchida T: Endobronchial ultrasound elastography in the diagnosis of mediastinal and hilar lymph nodes. Jpn J Clin Oncol 2014;44: 956-962.

. 
10 Nakajima T, Yasufuku K, Iyoda A, Yoshida S, Suzuki M, Sekine Y, Shibuya K, Hiroshima K, Nakatani Y, Fujisawa T: The evaluation of lymph node metastasis by endobronchial ultrasound-guided transbronchial needle aspiration: crucial for selection of surgical candidates with metastatic lung tumors. J Thorac Cardiovasc Surg 2007;134:1485-1490.

11 Rusch VW, Asamura H, Watanabe H, Giroux DJ, Rami-Porta R, Goldstraw P; Members of IASLC Staging Committee: The IASLC lung cancer staging project: a proposal for a new international lymph node map in the forthcoming seventh edition of the TNM classification for lung cancer. J Thorac Oncol 2009; 4:568-577.
2 Tournoy KG, Annema JT, Krasnik M, Herth FJ, van Meerbeeck JP: Endoscopic and endobronchial ultrasonography according to the proposed lymph node map definition in the seventh edition of the tumor, node, metastasis classification for lung cancer. J Thorac Oncol 2009;4:1576-1584.

13 Fujiwara T, Yasufuku K, Nakajima T, Chiyo M, Yoshida S, Suzuki M, Shibuya K, Hiroshima K, Nakatani Y, Yoshino I: The utility of sonographic features during endobronchial ultrasound-guided transbronchial needle aspiration for lymph node staging in patients with lung cancer: a standard endobronchial ultrasound image classification system. Chest 2010;138:641-647.
14 Nakajima T, Anayama T, Shingyoji M, Kimura H, Yoshino I, Yasufuku K: Vascular image patterns of lymph nodes for the prediction of metastatic disease during EBUS-TBNA for mediastinal staging of lung cancer. J Thorac Oncol 2012;7:1009-1014.

15 Inage T, Nakajima T, Yoshida S, Yoshino I: Endobronchial elastography in the evaluation of esophageal invasion. J Thorac Cardiovasc Surg 2015;149:576-577.

16 Nakajima T, Shingyouji M, Nishimura H, Iizasa T, Kaji S, Yasufuku K, Kimura H: New endobronchial ultrasound imaging for differentiating metastatic site within a mediastinal lymph node. J Thorac Oncol 2009;4:12891290. 